\title{
Biomedical Photopolymers in 3D Printing
}

\begin{abstract}
Purpose

$3 \mathrm{D}$ printing of acrylic-based medical devices is projected to grow exponentially despite the limitations of vat photopolymerization and the lack of information on the clinical performance of the materials. The purpose of this article is to address an issue of critical importance in the translation of the $3 \mathrm{D}$ printed structures to the clinic, which is assessing the toxicity of the polymers and their precursors.
\end{abstract}

\section{Design}

This review highlights the different manufacturing processes, challenges, and novel experimental work including the zebrafish embryo model which offers a potential method for toxicity profiling of biomedical photopolymers and their precursors due to its high genetic similarity to humans.

\section{Findings}

Materials information and experimental data available so far suggest that there is a need for regular and rigorous evaluation of new materials to establish their safety and protect users engaging in biomedically-related 3D printing activities.

\section{Originality}

The review identifies stringent, contemporary, and cost-effective analytical methods for assessing the safety of biomedical photopolymers and their precursors.

\section{Keywords}

Photopolymer; Photopolymerization; 3D Printing; Zebrafish embryo model; Medical devices; Biomaterials. 


\section{Introduction}

Although natural polymers such as proteins, cellulose, silk and natural rubber have existed since the dawn of time, it was the revolutionary discovery of synthetic polymers in the nineteenth century that led to plastics which have become an essential element of modern life (Fried, 2014). The word "polymer" is derived from Greek: "polys" and "meros" meaning "many" and “part”, respectively (Jensen, 2008). Polymers are identified by the structural and repeating units in their chains. A structural unit in a polymer chain represents a residue from a monomer used in the synthesis of the polymer, whereas a repeating unit refers to a structural unit or a covalently bonded combination of two complementary structural units, which is repeated many times to make the whole chain (Mandal, 2014). In general, polymers are molecules composed of repeating units connected by covalent bonds in a variety of ways (Ravve, 2012). Photopolymers, on the other hand, are polymeric materials that change their structural and chemical properties when exposed to light, usually within the ultraviolet $(100-400 \mathrm{~nm})$ and visible light $(400-740 \mathrm{~nm})$ region of the electromagnetic spectrum (Chua et al., 2017) by a process known as photopolymerization or photoinitiated polymerization.

\section{Photopolymerization in 3D Printing}

Photopolymerization is considered to be the most effective way to transform solvent-free resins into solid polymers, at ambient temperature (Decker, 1996). Compared to thermally-activated polymerization, it is more economical and offers a myriad of practicalities in imaging, microelectronics, graphic arts, printing plates, photoresists, laser direct imaging, computer-toplate technology, holographic optical elements and dentistry, to name a few (Tehfe et al., 2013). In $3 \mathrm{D}$ printing (3DP), the capacity to use photopolymerization to initiate speedy polymerization of solvent-free liquid resins accounts for its utility in the manufacturing of medical devices. 3DP simply involves importing a virtual model (usually "STL" file) into a designated 3D printer 
to build parts in successive (two-dimensional) layers until the desired 3D part is completed (Alifui-Segbaya et al., 2017b). 3DP also comprises a host of processes and technologies that offer a diverse spectrum of capabilities for the manufacturing of parts, end-use products and medical devices using different materials (3d Printing Industry, 2015). For this review, stereolithography, digital light processing and material jetting being photopolymerization processes currently used for acrylic-based medical devices are discussed. The complete manufacturing process is discussed in detail elsewhere in literature (Stansbury and Idacavage, 2016, Lee et al., 2017, Ligon et al., 2017).

\subsection{Stereolithography}

Stereolithography (SL) is considered the pioneer of 3DP processes (Hull, 1986, 3d Systems, 2017a). In a traditional SL system shown in Figure 1A (3d Printing and Additive Manufacturing, 2017), layers of liquid photopolymer resin from a vat are selectively cured or solidified with ultraviolet (UV) laser beam to form physical parts. For each printing step, the laser scans across the surface of the resin and solidifies it in predetermined $x-y$ directions, leaving the surrounding area in a liquid form. The build platform in the vat then drops fractionally (z-direction), submerging the solidified layer into the resin to be recoated and irradiated (3d Systems, 2015). In an inverted SL system (Figure 1B), the incident light is emitted from the bottom of the vat to solidify the liquid resin. The penetration depth i.e., photosensitive energy that goes into the resin is dependent on the type of photoinitiator, concentration of photoinitiator, and wavelength of the laser. The cure depth defines the thickness of each solidified layer and is influenced by the irradiation dose, critical energy and concentration of the resin (Tran and Wen, 2014). In general, the functionality of parts manufactured by SL is controlled by parameters that include physicochemical properties of the resins, speed and resolution of the optical scanning systems, the power, wavelength and types 
of laser used, the spot size of the laser, the recoating system and the postprocessing steps (Chua and Leong, 2015).
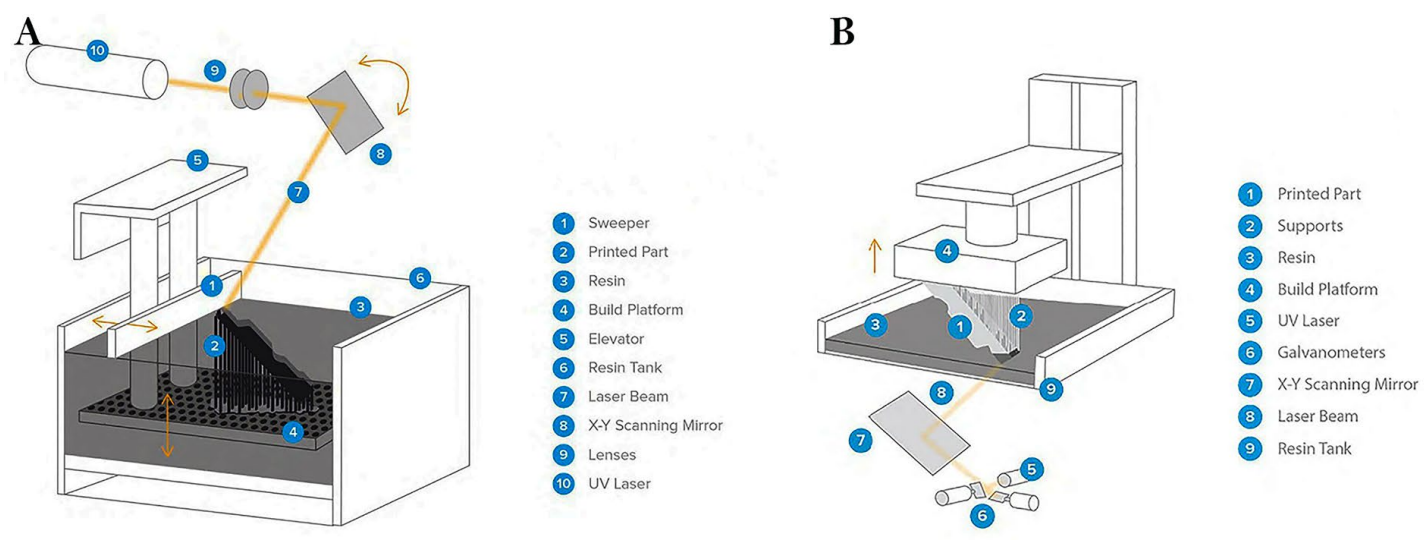

Figure 1 Schematic of traditional (A) and inverted (B) stereolithography 3D printers. Republished with permission of Mary Ann Liebert, Inc., New Rochelle, NY, from Expanding 3D printing technologies to high-volume applications and beyond, 3D Printing and Additive Manufacturing, eBook, 2017.

\subsection{Digital light processing}

Digital light processing (DLP) is similar to SL in that both are vat photopolymerization processes that require washing built parts in organic solvents to remove wet resin remnants, followed by postcuring in a UV oven to harden them. DLP, however, uses a more conventional light source such as an arc lamp, with a liquid crystal display panel or a deformable mirror device, which is applied to the entire surface of the vat of resin in a single pass, relatively

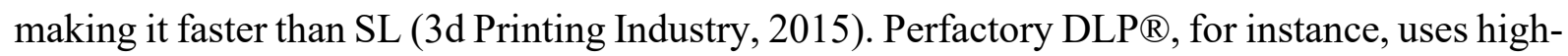
definition projectors that deliver light in pixels, creating volumetric pixels or voxels in the resin. The projector emit light in multiple intensities along a spectrum that goes from white to grey and black, thus enabling parts to be built in varying depths at fixed $\mathrm{x}-\mathrm{y}$ dimensions (Envisiontec Gmbh, 2016). The newly introduced continuous liquid interface production (CLIP), combines 
DLP technology with oxygen permeable optics and programmable resins to build 3D parts: incident light is projected through oxygen-permeable window ("dead zone") to selectively cure layers of resin flowing continuously above it (Carbon Inc.).

\subsection{Material Jetting}

In material jetting (MJ), liquid photopolymer resin is selectively squirted through multiple jet heads, and then cured with a passing of UV light as each layer is deposited. The jet heads are fixed (in $x-y$ directions) whereas the build tray is lowered (z-direction) for resin to be added and solidified. MJ also allows for simultaneous deposition of materials with multi-coloured attributes and physical properties. Depending on the technology, support structures can be melted away in a heated oven before cleaning (3d Systems, 2017b) or cautiously removed with a water jet or by hand (Stratasys, 2015). Unlike SL and DLP, no post-curing is required for MJ parts.

\section{Basic chemistry of phopolymerization}

Acrylics are probably the most versatile family of monomers that can be used to prepare polymers with rigid, flexible, ionic, nonionic, hydrophobic, or hydrophilic properties (Merck, 2017). They are preferred in free-radical polymerization (FRP) because of the high reactivity of the acrylate double bond. Under intense illumination, crosslinking polymerization of resins proceeds extensively within a fraction of a second to generate a three-dimensional polymer network. The speedy polymerization of the resins occurs by virtue of aromatic ketone photoinitiators undergoing fast homolytic cleavage upon irradiation (Decker, 1996, Decker, 2002). Liquid photopolymer resins for 3DP of medical devices (e.g., Figure 2 (Alifui-Segbaya, 2018)) are largely proprietary but are usually composed of photoinitiators, mono-ormultifunctional monomers and functionalized oligomers. The photoinitiators absorb the incident light and generate reactive radicals or ions; mono-or-multifunctional monomers act as 
reactive diluents to adjust viscosity; and functionalized oligomers constitute the backbone of the polymer after polymerization (Decker, 2002). Monofunctional monomers in the resins, can either act as a crosslinker or reactive diluent to adjust formulation viscosity while multifunctional monomers act concurrently as a crosslinker and diluent (Pandey, 2014).

Polymerization reaction consists of three dinstinct stages: initiation, propagation and termination. Initiation involves the formation of radicals that react with vinyl monomers. Propagation describes the rapid and progressive addition of monomers to growing polymer chain without changes to active centers. Termination occurs when free-radical chain reactions end either by combination (e.g., styrene) or disproportionation (e.g., methyl methacrylate). By combination, two growing polymer chains react with each other to form a single nonreactive polymer whereas by disproportionation, a hydrogen atom is transferred from one radical to another to form polymers with saturated and unsaturated ends. Additionally, a transfer of a growth active site from active chain to a previously inactive one might occur (Polymer Properties Database, 2018). To illustrate, the photoinitiator (PI) generates free radicals (PI $\rightarrow$ $\left.\mathrm{PI}^{*}(h v) \rightarrow 2 \mathrm{R} \bullet\right)$, which are then transferred to active groups on the monomer $(\mathrm{M} \bullet)$ chains $(\mathrm{R} \bullet$ $+\mathrm{M} \rightarrow \mathrm{RM} \bullet$ ) to form a crosslinked phase in repeated addition $(\mathrm{RM} \bullet)_{\mathrm{n}} \rightarrow$ polymer) (Fouassier et al., 2010). For enhanced clinical outcomes, resins should possess a high curing rate, good storage stability, low viscosity, low toxicity, and display adequate mechanical properties after polymerization (Li et al., 2016). Resins with relatively low viscosity are likely to produce rapid polymerisation yielding crosslinked polymers with properties suited to the demands imposed by the target application (Vitale and Cabral, 2016). The mechanical properties of photocured materials thus depend primarily on the chemical structure, functionality and concentration of the various constituents of the resin, and the degree of cure (Decker, 2002). Despite the high reactivity of acrylate double bond, oxygen inhibition due to excited triplet state quenching by 
$\mathrm{O}_{2}$ and scavenging of initiating $\mathrm{R} \bullet$, as well as propagating $\mathrm{RM}_{\mathrm{n}} \bullet$ radicals by $\mathrm{O}_{2}$ (Tehfe et al., 2013) occur as drawbacks of the FRP process. Likewise, the relatively low thermal resistance, glass transition temperature and physical properties of the resultant polymers (Crivello and Reichmanis, 2014).

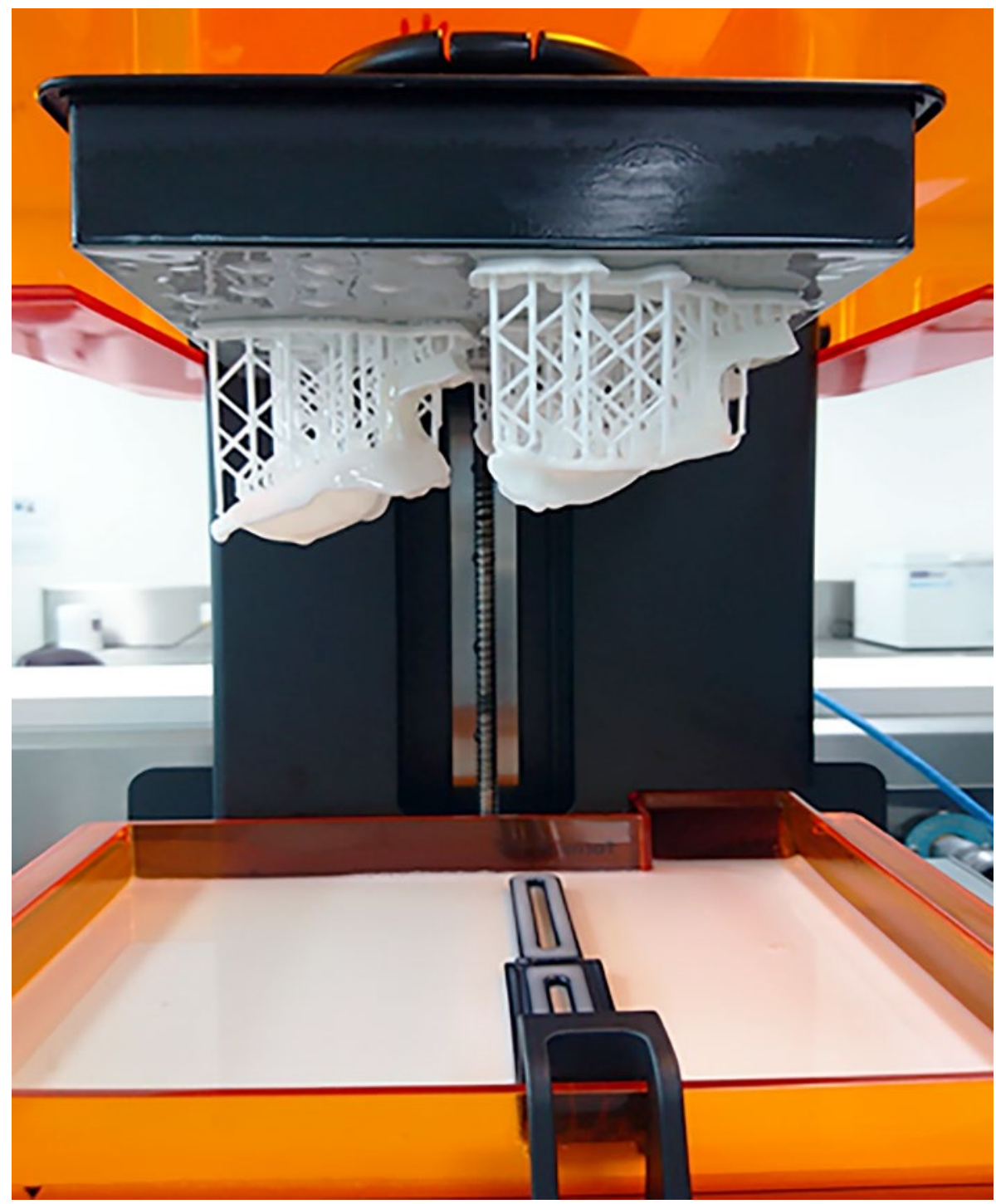

Figure 2 Custom impression trays built from a liquid photopolymer resin in "Form 2" inverted SL printer. Republished from Toxicological assessment of photopolymers in additive manufacturing using the innovative zebrafish embryo model, Alifui-Segbaya, F. PhD Thesis, Griffith University, 2018. 


\section{Biocompatibility of acrylic-based medical devices in 3D printing}

In FRP, polymerization of carbon-carbon double bond is critical since the carbonyl group is not prone to polymerization by radical initiators due to its polarized nature (Odian, 2004). Free radicals attack on the 'unsaturated' vinyl double bond ( $\pi$-bond) forms a single bond ( $\sigma$-bond) to another carbon and an unpaired electron (Kim and Watts, 2008). As polymerization progresses, the amount of aliphatic double bonds decreases but in practice, the process does not guarantee a 100\% monomer conversion rate (Duray et al., 1997). 3DP being an iterative process could also result in undesired material chemistries. The quality of additively manufactured parts may vary significantly regardless of whether they were manufactured using different 3D printers or with the same 3D printer and a standardised workflow of parameters, process steps, and materials (Food and Drug Administration, 2016). It is important to note that the dimensional accuracy of the parts is also of paramount importance, for instance, medical or anatomical models for diagnosis, surgical planning, and the reconstruction of posttraumatic defects, tumoral resections, and other complex craniofacial defects (Mazzoli et al., 2007).

Apart from the medical applications of acrylic materials, their widespread use also raises questions pertinent to their toxicological properties, and their short-and long-term health effects on persons exposed to them (Autian, 1975). For medical devices, it is recommended to use approved materials, apposite manufacturing parameters and postprocessing techniques to minimise the potential effects to residual monomer and degradation products (Alifui-Segbaya et al., 2018). Interestingly, disclaimers for some photopolymers state that, "it is the responsibility of the customer, its respective customers and end-users to determine the biocompatibility of all of the components, printed parts, and all other materials used in the finished product for their respective purposes, including prolonged skin contact (of more than 30 days) and short-term mucosal-membrane contact (of up to $24 \mathrm{~h}$ )" (Stratasys, 2016a, 
Stratasys, 2016b). Table 1 shows compositional differences between liquid and photocured resins; data for the latter were obtained in-house using gas chromatography - mass spectrometry (GC-MS) protocols (Alifui-Segbaya and George, 2018). Also, the gradations of toxicity (Figure 3) elicited by the photocured materials in zebrafish bioassays (Alifui-Segbaya et al., 2017a) were possibly influenced by their physicochemical characteristics, before and after immersion in ethanol (Alifui-Segbaya et al., 2018). 
Table 1 Composition of liquid and photocured ${ }^{l}$ biomedical photopolymers

MED610 Liquid resin (w/w \%): 20-30\% Exo-1,7,7-trimethylbicyclo [2.2.1] hept-2-yl acrylate; 5-10\% Tricyclodecane dimethanol diacrylate and $\approx 60 \%$ is proprietary.

Photocured resin ${ }^{2}$ : 2-Propanol, 1-methoxy-; Cyclohexanone; Benzaldehyde; 2-Propyl-1-pentanol and 2-Propenoic acid, 1,7,7-trimethylbicyclo [2.2.1] hept-2-yl ester, exo-

Photocured resin with ethanol treatment: 2-Propanol, 1-methoxy-; Cyclohexanone; Benzaldehyde; Cyclohexene, 1-methyl-4-(1-methylethenyl)-; Linalool and 2-Propenoic acid, 1,7,7-trimethylbicyclo [2.2.1] hept-2-yl ester, exo-

MED620

Liquid resin (w/w \%): $<30 \%$ Acrylic monomer; $<25 \%$ Exo-1,7,7 Trimethylbicyclo [2.2.1] hept 2-yl acrylate $;<15 \%$ Acrylic oligomer; $<3 \%$ Photoinitiator; $<0.8 \%$ Titanium dioxide; $<0.3 \%$ Acrylic acid ester; the rest is proprietary.

Photocured resin 3: 2-Propanol, 1-methoxy-; Acetic acid, butyl ester; Ethylbenzene; Benzene, 1,3-dimethyl-; Cyclohexanone; Bicyclo[3.1.1] heptane, 6,6-dimethyl-3-methylene-; Benzaldehyde; 2-Vinylfuran; Benzene, 1-ethyl2-methyl-; Pentanedioic acid, dimethyl ester; 2-Oxepanone; Isoborneol; N-Acryloylmorpholine; 2-Propenoic acid, 1,7,7-trimethylbicyclo [2.2.1] hept-2-yl ester, exo- and Furan, 2-butyltetrahydro-

Photocured resin with ethanol treatment: 2-Propanol, 1-methoxy-; Ethylbenzene; p-Xylene; Cyclohexanone; Benzaldehyde and 2-Propenoic acid, 1,7,7-trimethylbicyclo [2.2.1] hept-2-yl ester, exo-

Visijet SL Clear

Liquid resin (w/w \%): 60-75\% 4,4' Isopropylidenedicyclohexanol oligomeric reaction products with 1-chloro-2,3 epoxy propane; $15-25 \%$ 3-ethyl-3-hydroxymethyl-oxetane and Mixture containing triarylsulfonium salt: $50 \%$ propylene carbonate and 50\% mixed triarylsulfonium salts (1-5\%).

Photocured resin ${ }^{4}$ : Methyl Isobutyl Ketone; Cyclohexanone; Benzaldehyde; Propylene Carbonate; 1-Hexanol, 2ethyl-; 1,4-Dioxaspiro (4.5) decane-2-one; 2-Propenoic acid, 1,7,7-trimethylbicyclo [2.2.1] hept-2-yl ester, exo- and Methanone, (1-hydroxycyclohexyl) phenyl-

Photocured resin with ethanol treatment: 2-Propanol, 1-methoxy-; Methyl Isobutyl Ketone; Cyclohexanone; Ethanol, 2-butoxy-; Benzaldehyde; Propylene Carbonate; 1,4-Dioxaspiro (4.5) decane-2-one; 2-Propenoic acid, 1,7,7-trimethylbicyclo [2.2.1]hept-2-yl ester, exo- and Methanone, (1-hydroxycyclohexyl)phenyl-

Visijet M3 Crystal Liquid resin (w/w \%): 15-25\% Ethoxylated bisphenol A diacrylate; 14-24\% Urethane acrylate oligomers and 5$11 \%$ Tripropyleneglycol diacrylate.

Photocured resin 5: 2-Propanol, 1-methoxy-; Toluene; Cyclopentanol, 2-methyl; Cyclohexanone; Ethanol, 2butoxy-; Benzaldehyde; 2-Propyl-1-pentanol; 2-Oxepanone; 2-Propenoic acid, 1,7,7-trimethylbicyclo [2.2.1] hept2-yl ester, exo-; Tetrahydro [2,2'] bifuranyl-5-one and Methanone, (1-hydroxycyclohexyl) phenyl-

Photocured resin with ethanol treatment: 2-Propanol, 1-methoxy-; Toluene; Cyclopentanol, 2-methyl-, cis; Cyclohexanone; Ethanol, 2-butoxy-; Benzaldehyde; 1-Hexanol, 2-ethyl-; 2-Oxepanone; 2-Propenoic acid, 1,7,7trimethylbicyclo[2.2.1]hept-2-yl ester, exo; Furan, 2-butyltetrahydro- and Methanone, (1hydroxycyclohexyl)phenyl-

\footnotetext{
${ }^{1}$ Analysed by headspace GC-MS: Samples were frozen in liquid nitrogen at $-196^{\circ} \mathrm{C}$, ground into powder and tested in GCShimadzu TQ8040 GC-MS/MS (Shimadzu Corporation, Tokyo, Japan). The GC column used was Agilent J\&W DB5-MS 30m $0.25 \mathrm{~mm}$ ID $0.25 \mathrm{um}$ film thickness. Test parameters are, column oven temperature at $40.0^{\circ} \mathrm{C}$, injection temperature at $250{ }^{\circ} \mathrm{C}$, column flow rate at $1.16 \mathrm{~mL} / \mathrm{min}$, split ratio of 5.0 and a total run time of 15 minutes.

${ }^{2}$ Photocured MED610 was built by Stratasys (C-BONS International Center 108, Hong Kong, China) using Objet Eden260VS $3 \mathrm{D}$ printer.

${ }^{3}$ Photocured MED620 was built by Stratasys (C-BONS International Center 108, Hong Kong, China) using Objet Eden260VS $3 \mathrm{D}$ printer.

${ }^{4}$ Photocured Visijet SL Clear was built by 3D Systems (3D Systems, Rock Hill, SC) using ProJet 7000 HD printer parameters.

${ }^{5}$ Photocured Visijet M3 Crystal was built by (3D Systems, Rock Hill, SC) using MJP 3600 3D printer.
} 


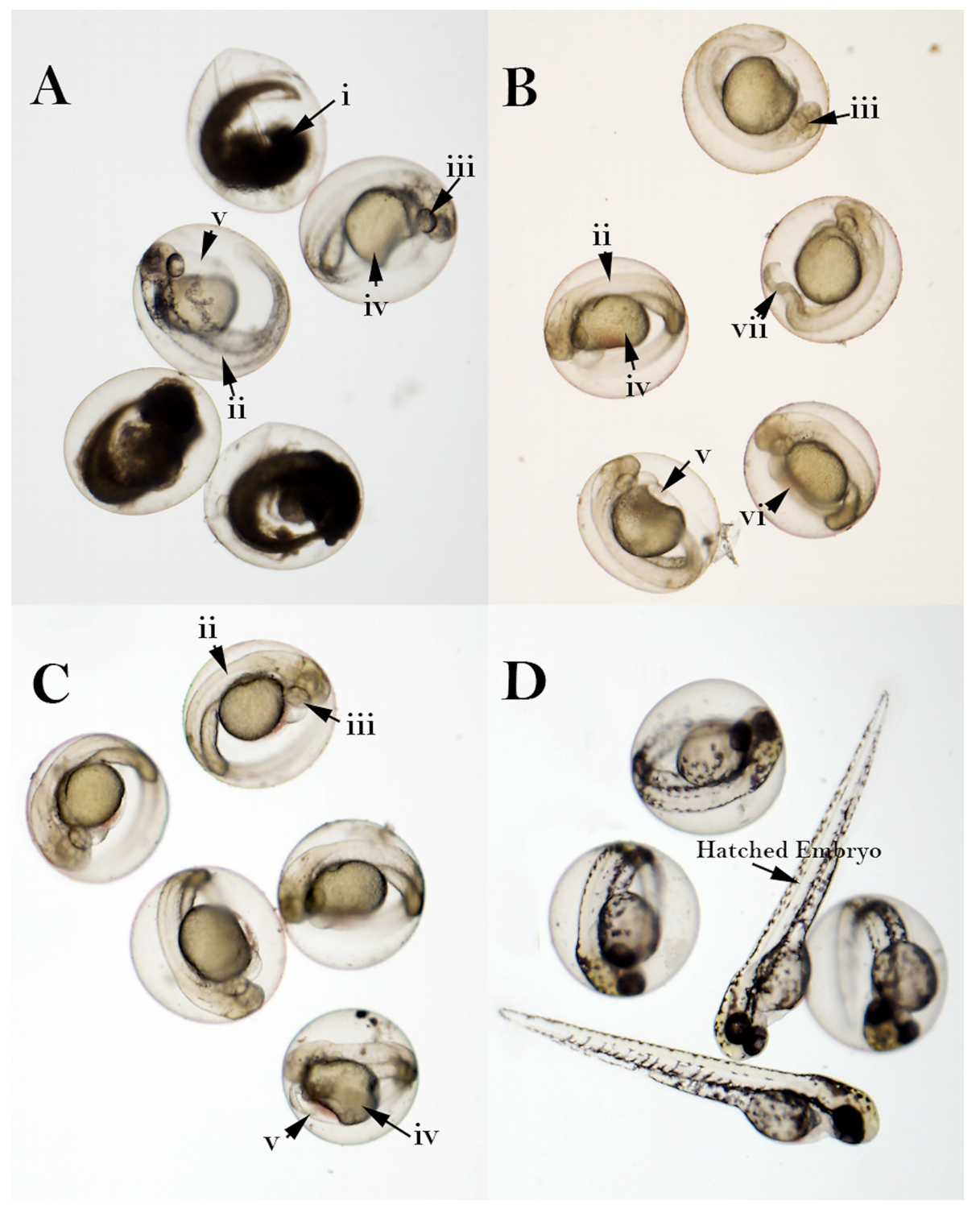

Figure 3 Zebrafish (AB/Tü) bioassays containing a: photocured and ethanol-treated visijet clear b: photocured visijet crystal c: photocured and ethanol-treated visijet crystal and d: control. The toxicity endpoints in "a", "b" and "c" are i mortality ii hypopigmented body iii hypopigmented eye iv deformed yolk v pericardial edema vi blood pooling vii. malformed tail. Republished with permission of Mary Ann Liebert, Inc., New Rochelle, NY, from Biocompatibility of Photopolymers in 3D Printing, Alifui-Segbaya, F., et al., Volume 4, Number 4, 2017; permission conveyed through Copyright Clearance Center, Inc. 


\section{Zebrafish as an alternative animal model for evaluating biocompatibility}

Since it is generally accepted that no biomaterial is $100 \%$ safe (Wataha, 2012), toxicological tests are designed in order to help the identification of toxicity mechanisms of chemical compounds, damage that may ensue in their exposure, and the necessary preventive measure to ameliorate their adverse effects (United States National Library of Medicine, 2004). Several tests are recommended by the International Organization for Standardization (ISO) based on intended use of materials or devices (Iso 7405, 2008). To determine acute toxicity, in vitro cytotoxicity assays rely on immortalized cell lines that have little metabolic capability and often lack information on organ-specific or cell-type specific physiology (Committee on Predictive Toxicology Approaches for Military et al., 2015). Animal tests, on the other hand, are expensive, complex, and present laborious administrative challenges (Wataha, 2012). In this regard, zebrafish (non-mammalian in vivo) bioassay offers economy and ease of quantifying multiple toxicity endpoints, dose-response relationship, and toxicodynamics of chemicals or leachable substances (Hill et al., 2005, Strähle et al., 2012).

The use of zebrafish (Danio rerio) as a laboratory animal was pioneered by George Streisinger and others (Streisinger et al., 1981). Zebrafish are small benthopelagic cyprinid fish that originates from the Ganges River system, Burma, the Malakka peninsula and Sumatra. It has an average adult length ranging between 3 and $5 \mathrm{~cm}$, and an approximate generation time of 3 to 4 months at $26^{\circ} \mathrm{C}$ in both soft and hard waters. As an aquatic vertebrate species, it is easily obtainable and spawned, and under appropriate conditions will provide many non-adherent and transparent eggs (Braunbeck and Lammer, 2006, Belanger et al., 2010). Despite the physiological differences between fish and humans, $70 \%$ of protein-coding human genes are related to genes found in zebrafish. Likewise, $84 \%$ of genes known to be associated with human disease have a zebrafish counterpart (Howe et al., 2013). These similarities make zebrafish 
appropriate for research in cancer aetiology (Pei and Strauss, 2013), developmental biology, embryogenesis, pharmaceutical drug discovery (Detrich et al., 1999, Delvecchio et al., 2011, Kari et al., 2007, Gustafson et al., 2012) and assessing teratogenic effects (Sipes et al., 2011, Selderslaghs et al., 2012), to name but a few.

The Organization for Economic Cooperation and Development (OECD) fish embryo toxicity (FET) test designed to determine acute toxicity of chemicals on embryonic stages of zebrafish (Oecd Test Guideline 236, 2013) has been successfully applied to toxicity profiling of photopolymers in 3DP (Oskui et al., 2016, Macdonald et al., 2016, Alifui-Segbaya et al., 2017a, Alifui-Segbaya and George, 2018, Alifui-Segbaya et al., 2018). The wild-type strain recommended for chemical screening is also popular for research related to sequencing, genetic screening, gene expression and transgenesis (Haper and Lawrence, 2011). It is important to understand that, the FET test is not considered an animal experiment since fish in their early stage are "not sufficiently aware" that they will suffer when a procedure is carried out on them"(Braunbeck et al., 2015). Nonetheless, some developmental endpoints induced by additively manufactured methacrylates in fish bioassays are comparable to those reported in animal studies that linked methacrylic esters to embryonic fatal toxicity, teratogenicity, and cardiovascular function (Alifui-Segbaya et al., 2018). In general, the zebrafish is an excellent model for developmental toxicity and offers advantages such as external fertilization, high fecundity, transparent embryos, that permit 'whole organism' assessment through direct observation of internal organs and ease of phenotype assessment over other vertebrates or rodents models, in which aspects of organogenesis and disease pathology cannot be examined without interventions such as surgery or post-mortem examination (Lieschke and Currie, 2007). 


\section{Conclusions}

Currently, very little data is published on systematic studies of the biocompatibility of 3D printable polymers and their precursors despite the increasing popularity of 3DP and contemporaneous influx of new materials. Thus, there is a need for methods to stringently evaluate these materials to establish their safety and protect users engaging in biomedicallyrelated 3DP activities. In the long term, standards for preclinical evaluation could be revised to reflect the current trends in biomaterials, manufacturing techniques, and biological evaluation of medical devices and precursors they are generated from.

\section{Acknowledgement}

I would like to thank Objective 3D (Australia) and 3D Systems (Australia) for supplying printed materials to support my ongoing project. My gratitude also goes to Adam White and Jasper Bowman (School of Natural Sciences, Griffith University) for their assistance with GC-MS analysis of the materials presented in this review.

\section{References}

3D Printing and Additive Manufacturing (2017). Expanding 3D printing technologies to highvolume applications and beyond., Mary Ann Liebert, Inc. Publishers, available at: http://www.nxtbook.com/nxtbooks/mal/formlabsebook201709/index.php (accessed 6 June 2018).

3D Printing Industry (2015), "3D Printing Basics: The Free Beginner's Guide", available at: http://3dprintingindustry.com/wp-content/uploads/2014/07/3D-Printing-Guide.pdf (accessed 16 January 2016).

3D Systems (2015), "Stereolithography", available at: http://www.3dsystems.com/resources/information-guides/stereolithography/sla (accessed 26 November 2015).

3D Systems (2017a), "Our Story", available at: https://au.3dsystems.com/our-story (accessed 25 April 2017). 
3D Systems (2017b), "What is MJP (MultiJet Printing)?", available at: https://au.3dsystems.com/resources/information-guides/multi-jet-printing/mjp (accessed 10 March 2017).

Alifui-Segbaya, F. (2018). Toxicological assessment of photopolymers in additive manufacturing using the innovative zebrafish embryo model. Doctor of Philosophy, $\mathrm{PhD}$ Doctorate Thesis, Griffith University. URI: http://hdl.handle.net/10072/381385.

Alifui-Segbaya, F., Bowman, J., White, A. R., Varma, S., Lieschke, G. J. and George, R. (2018), "Toxicological assessment of additively manufactured methacrylates for medical devices in dentistry". Acta Biomaterialia, Vol. 78 No. pp. 64-77.

Alifui-Segbaya, F. and George, R. (2018), "Biocompatibility of 3D-Printed Methacrylate for Hearing Devices". Inventions, Vol. 3 No. 3, pp. 52.

Alifui-Segbaya, F., Varma, S., Lieschke, G. J. and George, R. (2017a), "Biocompatibility of Photopolymers in 3D Printing". 3D Printing and Additive Manufacturing, Vol. 4 No. 4, pp. $185-91$.

Alifui-Segbaya, F., Williams, R. J. and George, R. (2017b), "Additive Manufacturing: A Novel Method for Fabricating Cobalt-Chromium Removable Partial Denture Frameworks". European Journal of Prosthodontics and Restorative Dentistry, Vol. 25 No. 2, pp. 73-78.

Autian, J. (1975), "Structure-toxicity relationships of acrylic monomers". Environmental Health Perspectives, Vol. 11 pp. 141-52.

Belanger, S. E., Balon, E. K. and Rawlings, J. M. (2010), "Saltatory ontogeny of fishes and sensitive early life stages for ecotoxicology tests". Aquatic Toxicology, Vol. 97 No. 2, pp. 8895.

Braunbeck, T., Kais, B., Lammer, E., Otte, J., Schneider, K., Stengel, D. and Strecker, R. (2015), "The fish embryo test (FET): origin, applications, and future". Environmental Science and Pollution Research, Vol. 22 No. 21, pp. 16247-61.

Braunbeck, T. and Lammer, E. 2006. Fish embryo toxicity assay. Dessau, Germany: Organisation for Economic Co-operation and Development Publishing.

Carbon Inc. (2018), "Carbon: our process", available at: https://www.carbon3d.com/process/ (accessed 20 May 2018).

Chua, C. K. and Leong, F. K. (2015). 3D Printing and Additive Manufacturing: Principles and Applications, World Scientific Publishing Co. Pte. Ltd., Singapore.

Chua, C. K., Matham, M. V. and Kim, Y.-J. (2017). "Materials for laser-based 3D printing and manufacturing", in Lasers in 3D Printing and Manufacturing, World Scientific, Hackensack, pp. 49-91.

Committee on Predictive Toxicology Approaches for Military, Assessments of Acute Exposures, Board on Environmental Studies and Toxicology, Board on Life Sciences, Division on Earth and Life Studies and The National Academies of Sciences, E., and 
Medicine, (2015). Application of Modern Toxicology Approaches for Predicting Acute Toxicity for Chemical Defense, The National Academies Press, Washington, DC.

Crivello, J. V. and Reichmanis, E. (2014), "Photopolymer Materials and Processes for Advanced Technologies". Chemistry of Materials, Vol. 26 No. 1, pp. 533-48.

Decker, C. (1996), "Photoinitiated crosslinking polymerisation". Progress in Polymer Science, Vol. 21 No. 4, pp. 593-650.

Decker, C. (2002), "Kinetic Study and New Applications of UV Radiation Curing". Macromolecular Rapid Communications, Vol. 23 No. 18, pp. 1067-93.

Delvecchio, C., Tiefenbach, J. and Krause, H. M. (2011), "The zebrafish: a powerful platform for in vivo, HTS drug discovery". Assay and Drug Development Technologies, Vol. 9 No. 4, pp. 354-61.

Detrich, H. W., 3rd, Westerfield, M. and Zon, L. I. (1999), "Overview of the Zebrafish system". Methods in Cell Biology, Vol. 59 No. pp. 3-10.

Duray, S. J., Gilbert, J. L. and Lautenschlager, E. P. (1997), "Comparison of chemical analysis of residual monomer in a chemical-cured dental acrylic material to an FTIR method". Dental Materials, Vol. 13 No. 4, pp. 240-45.

EnvisionTec GmBH (2016), "Advanced DLP for superior 3D printing", available at: https://envisiontec.com/better3dprintingtechnology/ (accessed 5 June 2018).

Food and Drug Administration (2016). Technical Considerations for Additive Manufactured Devices: Draft Guidance for Industry and Food and Drug Administration Staff, Food and Drug Administration, Rockville (MD), available at: https://www.fda.gov/downloads/MedicalDevices/DeviceRegulationandGuidance/GuidanceDo cuments/UCM499809.pdf (accessed 3 June 2018).

Fouassier, J. P., Allonas, X., Lalevee, J. and Dietlin, C. (2010). "Photoinitiators for free radical polymerization reactions", in (Ed.), Photochemistry and Photophysics of Polymeric Materials, John Wiley \& Sons, Inc., Hoboken, NJ, pp. 351-419.

Fried, J. R. (2014). Polymer science and technology, Pearson Education, Inc., Upper Saddle River.

Gustafson, A. L., Stedman, D. B., Ball, J., Hillegass, J. M., Flood, A., Zhang, C. X., PanzicaKelly, J., Cao, J., Coburn, A., Enright, B. P., Tornesi, M. B., Hetheridge, M. and AugustineRauch, K. A. (2012), "Inter-laboratory assessment of a harmonized zebrafish developmental toxicology assay - Progress report on phase I". Reproductive Toxicology, Vol. 33 No. 2, pp. $155-64$.

Haper, C. and Lawrence, C. (2011). The Laboratory Zebrafish, CRS Press, Boca Raton, FL.

Hill, A. J., Teraoka, H., Heideman, W. and Peterson, R. E. (2005), "Zebrafish as a Model Vertebrate for Investigating Chemical Toxicity". Toxicological Sciences, Vol. 86 No. 1, pp. 619. 
Howe, K., Clark, M. D., Torroja, C. F., Torrance, J., Berthelot, C., Muffato, M., Collins, J. E., Humphray, S., et al. (2013), "The zebrafish reference genome sequence and its relationship to the human genome". Nature, Vol. 496 No. 7446, pp. 498-503.

Hull, C. W. (1986). Apparatus for production of three-dimensional objects by stereolithography. USA patent application US 06/638,905.

ISO 7405 2008. Dentistry - Evaluation of biocompatibility of medical devices used in dentistry. Geneva: International Organization for Standardization.

Jensen, W. B. (2008), "The Origin of the Polymer Concept". Journal of Chemical Education, Vol. 85 No. 5, pp. 624.

Kari, G., Rodeck, U. and Dicker, A. P. (2007), "Zebrafish: an emerging model system for human disease and drug discovery". Clinical Pharmacology \& Therapeutics, Vol. 82 No. 1, pp. $70-80$.

Kim, S.-H. and Watts, D. C. (2008), "Degree of conversion of bis-acrylic based provisional crown and fixed partial denture materials". The Journal of Korean Academy of Prosthodontics, Vol. 46 No. 6, pp. 639-43.

Lee, J.-Y., An, J. and Chua, C. K. (2017), "Fundamentals and applications of 3D printing for novel materials". Applied Materials Today, Vol. 7, pp. 120-33.

Li, J., Cui, Y., Qin, K., Yu, J., Guo, C., Yang, J., Zhang, C., Jiang, D. and Wang, X. (2016), "Synthesis and properties of a low-viscosity UV-curable oligomer for three-dimensional printing". Polymer Bulletin, Vol. 73 No. 2, pp. 571-85.

Lieschke, G. J. and Currie, P. D. (2007), "Animal models of human disease: zebrafish swim into view". Nature Reviews Genetics, Vol. 8 No. 5, pp. 353-67.

Ligon, S. C., Liska, R., Stampfl, J., Gurr, M. and Mülhaupt, R. (2017), "Polymers for 3D Printing and Customized Additive Manufacturing". Chemical Reviews, Vol. 117 No. 15, pp. 10212-90.

Macdonald, N. P., Zhu, F., Hall, C. J., Reboud, J., Crosier, P. S., Patton, E. E., Wlodkowic, D. and Cooper, J. M. (2016), "Assessment of biocompatibility of 3D printed photopolymers using zebrafish embryo toxicity assays". Lab on a Chip, Vol. 16 No. 2, pp. 291-97.

Mandal, B. M. (2014). Fundamentals of Polymerization, World Scientific Publishing Co Pte Ltd, Singapore.

Mazzoli, A., Germani, M. and Moriconi, G. (2007), "Application of Optical Digitizing Techniques to Evaluate the Shape Accuracy of Anatomical Models Derived From Computed Tomography Data". Journal of Oral and Maxillofacial Surgery, Vol. 65 No. 7, pp. 1410-18.

Merck (2017), "Acrylic monomers", available at: https://www.sigmaaldrich.com/materialsscience/material-science-products.html?TablePage=16397884 (accessed 3 December 2017).

Odian, G. (2004). Principles of polymerization, John Wiley \& Sons, Inc., Hoboken. 
OECD Test Guideline 236 2013. Fish Embryo Acute Toxicity Test. Paris: Organisation for Economic Co-operation and Development Publishing.

Oskui, S. M., Diamante, G., Liao, C., Shi, W., Gan, J., Schlenk, D. and Grover, W. H. (2016), "Assessing and Reducing the Toxicity of 3D-Printed Parts". Environmental Science \& Technology Letters, Vol. 3 No. 1, pp. 1-6.

Pandey, R. (2014). Photopolymers in 3D printing applications. Plastics Technology, Degree Thesis, Arcada University of Applied Sciences.

Pei, D.-S. and Strauss, P. R. (2013), "Zebrafish as a model system to study DNA damage and repair". Mutation Research/Fundamental and Molecular Mechanisms of Mutagenesis, Vol. 743-744, pp. 151-59.

Polymer properties database (2018), "Mechanism of free radical polymerization", available at: http://polymerdatabase.com/polymer\%20chemistry/radical\%20mechanism.html (accessed 24 May 2018).

Ravve, A. (2012). Principles of Polymer Chemistry, Springer, New York.

Selderslaghs, I. W. T., Blust, R. and Witters, H. E. (2012), "Feasibility study of the zebrafish assay as an alternative method to screen for developmental toxicity and embryotoxicity using a training set of 27 compounds". Reproductive Toxicology, Vol. 33 No. 2, pp. 142-54.

Sipes, N. S., Padilla, S. and Knudsen, T. B. (2011), "Zebrafish-As an Integrative Model for Twenty-first Century Toxicity Testing". Birth Defects Research Part C-Embryo TodayReviews, Vol. 93 No. 3, pp. 256-67.

Stansbury, J. W. and Idacavage, M. J. (2016), "3D printing with polymers: Challenges among expanding options and opportunities". Dental Materials, Vol. 32 No. 1, pp. 54-64.

Strähle, U., Scholz, S., Geisler, R., Greiner, P., Hollert, H., Rastegar, S., Schumacher, A., Selderslaghs, I., Weiss, C., Witters, H. and Braunbeck, T. (2012), "Zebrafish embryos as an alternative to animal experiments: A commentary on the definition of the onset of protected life stages in animal welfare regulations". Reproductive Toxicology, Vol. 33 No. 2, pp. 12832 .

Stratasys (2015), "PolyJet Technology; 3D print with precision in a wide range of materials", available at: http://www.stratasys.com/3d-printers/technologies/polyjet-technology (accessed 22 October 2015).

Stratasys (2016a), "Printing Bio-compatible Parts on PolyJet ${ }^{\mathrm{TM}}$ 3D Printers with MED610TM", available at:

http://usglobalimages.stratasys.com/Main/Files/MSDS/MED610_Usage_Terms.pdf?v=63588 8173072389415 (accessed 20 June 2018).

Stratasys (2016b), "Printing Bio-compatible Parts on PolyJet ${ }^{\mathrm{TM}}$ 3D Printers with MED620", available at:

http://usglobalimages.stratasys.com/Main/Files/SDS/MED620_Usage_Terms_1116.pdf?v=63 6160085940019299 (accessed 18 January 2018). 
Streisinger, G., Walker, C., Dower, N., Knauber, D. and Singer, F. (1981), "Production of clones of homozygous diploid zebra fish (Brachydanio rerio)". Nature, Vol. 291, pp. 293.

Tehfe, M., Louradour, F., Lalevée, J. and Fouassier, J.-P. (2013), "Photopolymerization Reactions: On the Way to a Green and Sustainable Chemistry". Applied Sciences, Vol. 3 No. 2, pp. 490-514.

Tran, V. and Wen, X. (2014). "Rapid prototyping technologies for tissue regeneration", in Narayan, R. (Ed.), Rapid Prototyping of Biomaterials, Woodhead Publishing, pp. 97-155.

United States National Library of Medicine (2004), "Toxicology", available at: https://www.nlm.nih.gov/tsd/acquisitions/cdm/subjects99.html (accessed 12 January 2018).

Vitale, A. and Cabral, J. T. (2016), "Frontal Conversion and Uniformity in 3D Printing by Photopolymerisation". Materials, Vol. 9 No. 9, pp. 760.

Wataha, J. C. (2012), "Predicting clinical biological responses to dental materials". Dental Materials, Vol. 28 No. 1, pp. 23-40.

\section{Author's Biography}

Frank Alifui-Segbaya is a Lecturer at the School of Dentistry and Oral Health, Griffith University. He graduated from the University of Wales Institute, Cardiff (now Cardiff Metropolitan University) with a BSc in Dental Technology with First Class Honours, completed an MPhil at the same university, and a PhD at Griffith University. Frank qualified as a dental technologist in 2005 and has served in other capacities: as an academic and a research scientist in Ghana, Germany, Wales, England and Australia. His research interests include biomaterials, zebrafish toxicology, and biocompatibility of dental devices, additive manufacturing, and the application of CAD/CAM to dentistry. Frank Alifui-Segbaya can be contacted at: f.alifuisegbaya@griffith.edu.au 